\title{
Escala de Aprendizajes Percibidos en la experiencia curricular «Programas de Intervención en Psicología»: evidencias psicométricas
}

\section{Scale of Perceived Learnings in the Curricular Experience «Intervention Programs in Psychology»: Psychometrics evidences}

\author{
Marivel Teresa Aguirre-Morales ${ }^{\mathrm{a}, *}$ \\ Lizley Janne Tantaleán-Terrones de Callohuanca ${ }^{\mathrm{b}, * *}$ \\ aUniversidad de San Martín de Porres, Perú \\ bUniversidad Nacional Federico Villarreal, Perú
}

\section{Resumen}

Este estudio presenta las evidencias psicométricas de la Escala de Aprendizajes Percibidos (32 reactivos), basada en los elementos didácticos y pedagógicos del proceso de enseñanza-aprendizaje de una Experiencia Curricular (EC) en formulación y ejecución de «Programas de Intervención en Psicología». Investigación instrumental-psicométrica, de enfoque cuantitativo-transversal, con recolección de datos post-EC, en 117 estudiantes universitarios. Su contraste estadístico se realizó en el entorno R para Análisis Factorial Confirmatorio y Análisis Factorial de Componentes Principales, entre otros. Los resultados indican un adecuado ajuste orientado a 5 factores $\left(\chi^{2}=130.755, g l=454, p=1.00, \mathrm{GFI}=.999, \mathrm{CFI}=1.00, \mathrm{TLI}=1.00, \mathrm{NNFI}=\right.$ $1.00, \mathrm{RMSEA}=.00, \mathrm{SRMR}=.065, \mathrm{RMR}=.037)$, ratificado en análisis paralelos. Se concluye en la conformación de un constructo robusto, fiable y válido, que explora la conciencia de los aprendizajes en materia de promociónprevención en salud, y susceptible de adaptación.

Palabras clave: escala, aprendizajes percibidos, programas de intervención en psicología, estudiantes universitarios.

Este es un artículo Open Access bajo la licencia Creative Commons Atribución-NoComercial-Compartirlgual 4.0

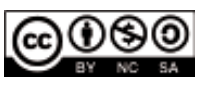




\begin{abstract}
This study presents the psychometric evidence of the Perceived Learnings Scale (32 items), based on the didactic and pedagogical elements of the teaching-learning process, of a Curricular Experience (CE) in the formulation and execution of «Intervention Programs in Psychology». Instrumentalpsychometric research, with a quantitative-transversal approach, with postEC data collection, in 117 university students. Its statistical contrast was performed in the $\mathrm{R}$ environment for Confirmatory Factor Analysis and Principal Component Factor Analysis, among others. The results indicate an adequate fit oriented to 5 factors $\left(\chi^{2}=130.755, g l=454, p=1.00\right.$, GFI $=$ $.999, \mathrm{CFI}=1.00, \mathrm{TLI}=1.00, \mathrm{NNFI}=1.00, \mathrm{RMSEA}=.00, \mathrm{SRMR}=.065, \mathrm{RMR}$ $=.037$ ), ratified in parallel analyzes. It concludes in the formation of a robust, reliable and valid construct that explores the awareness of learning in matters of promotion-prevention in health, and susceptible to adaptation.
\end{abstract}

Keywords: scale, perceived learnings, intervention programs in psychology, university students.

\title{
Introducción
}

La educación superior asume desafíos de forma continua y el contexto actual aceleró su capacidad de respuesta; más aún en profesiones ligadas a las ciencias de la salud, dada la naturaleza de sus Experiencias Curriculares (EC) y el riesgo de no continuidad de los estudiantes ante el reto de la virtualidad en el desarrollo de actividades que implican atención a usuarios.

Asimismo, la digitalización de contenidos profundizó las desigualdades de conectividad, mientras que los requerimientos de transformación del modelo educativo exigen docentes mentores, capaces de fomentar la investigación y el compromiso social en los estudiantes (Rosario et al., 2021); con mayor énfasis en carreras de servicio donde esta condición es inherente.

El bienestar mental de los individuos complementa la salud integral, habiéndose visibilizado su importancia con mayor énfasis ante las consecuencias de la pandemia, con un incremento moderado de trastornos 
de depresión y estrés, incluso en profesionales de la salud (HuarcayaVictoria, 2020). Motivo por el cual una intervención oportuna del especialista genera efectos favorables en la población desde los enfoques preventivos y promocionales, materializados en psicoeducación dispuesta a redireccionar pensamientos y emociones hacia comportamientos adaptativos.

En este sentido, en la formación del psicólogo se valoran los procesos que contribuyen a una correcta gestión del estudiante en sus intervenciones (diseño, implementación y ejecución), con un enfoque científico como fundamento formativo; es decir, saberes, destrezas y comportamientos que confluyan en aprendizajes efectivos. Según Quevedo et al. (2020), esta última competencia presentaría mayores dificultades ante la virtualidad, hecho que limita los alcances en investigación.

El aprovechamiento de una EC se asocia al ejercicio de la diada didácticopedagógica, más aún cuando su registro pretende trascender la calidad de los productos de aprendizaje. Por ello, si en lo didáctico el estudiante se nutre de saberes conceptuales, destrezas prácticas e interioriza el valor de la EC, en lo pedagógico el actor principal es el docente, dando vida a los procesos didácticos desde su saber transmitir: 1) conocimientos, 2) su aplicación y 3) profesionalismo. «Un docente gestor de saberes», planificador, metodológico y constructivista; promotor de actividades colaborativas y cooperativas, formulador de problemas, acompañante en la elaboración de productos mediante el monitoreo, la supervisión y la evaluación; todo ello con el objetivo de que el futuro profesional sea consciente de sus saberes y promueva su superación continua (Rodríguez, 2017).

La efectividad de una EC en el aprendizaje del estudiante universitario suele medirse en función de su rendimiento académico (calificaciones), como paradigma vigente desde la investigación respecto al «entorno educativo» (Rojas-Bolívar et al., 2021), el «clima en el aula» (León et al., 2021), los «factores institucionales percibidos» (Echeverría-Ramírez y Mazzitelli, 2021) y «la autorregulación del uso de dispositivos móviles en educación superior» (Romero-Rodríguez et al., 2021); logrando identificar 41 artículos publicados al mes de septiembre del 2021, según la base de datos Scopus. Una evidencia que parte del criterio del docente, mas no del dominio del estudiante, quien es el protagonista y principal beneficiario de la EC. 
En este contexto, la percepción del estudiante adquiere importancia, en especial desde el enfoque de competencias, para determinar los logros de aprendizaje en cada nivel (como efecto de evaluación de la asignatura), con énfasis en procesos complejos para generación de valor en la EC virtual. Como antecedente en la práctica educativa se considera la última validación psicométrica del Cuestionario de Jeffries (2005), que evalúa la percepción, satisfacción y resultados de aprendizaje en un escenario de simulación clínica (Arancibia et al., 2020); la validez de instrumentos de percepción de aprendizaje en la calidad, características, ventajas y satisfacción con el proceso formativo (Carpio et al., 2021); la valoración de asignaturas de educación física con un modelo dimensional que incluye: práctica, autoenseñanza, autoevaluación, experiencia, exploración libre, enseñanza recíproca, resolución de problemas, etc... (Espada et al., 2021); el diseño y validación de un cuestionario sobre percepción de aprendizaje basado en proyectos (Carrasco et al., 2015) y la adaptación de este instrumento a 40 reactivos que miden la condición sociodemográfica, las competencias técnicas-metodológicas-participativas-personales; así como la contribución del aprendizaje basado en problemas (Gil-Galván, 2018).

La asignatura asociada a la intervención preventiva y promocional en Salud Mental (SM) parte de su comprensión conceptual y operativa desde su implementación como política pública, la investigación en materia epidemiológica (prevalencia e incidencia de problemáticas en SM) y la selección de instrumentos. Desde lo práctico incluye tres momentos: 1) el diagnóstico situacional (línea base) -el desarrollo de matrices analíticas- y el diseño de la propuesta de intervención; 2) la ejecución-monitoreoevaluación (con resultados que evidencien su efectividad); y 3) la elaboración del informe final; detalles que son sensibles a la percepción de adquisición de competencias conceptuales, procedimentales y actitudinales. Si a ello se añade la percepción del rol docente, la valoración del constructo de la asignatura y el nivel de satisfacción con la EC se puede configurar un proceso de evaluación consistente.

Para tal efecto, la presente investigación tuvo por objeto evaluar las evidencias psicométricas de la Escala de Aprendizajes Percibidos, para lo cual se planteó: a) analizar su índice de ajuste como modelo confirmatorio, b) analizar sus cargas factoriales, c) analizar los índices de discriminación 
según factor, d) diferenciar el comportamiento de los ítems (según población participante), e) analizar las medidas de consistencia interna según factor; y f) determinar el modelo confirmatorio con sus respectivas correlaciones internas.

Por tanto, la creación de un modelo de evaluación para una asignatura compleja genera valor en el proceso de la instrucción superior; más aún en un contexto virtual que exige capacidad de adaptación de las actividades en los diferentes ámbitos formativos. De tal modo, este estudio presenta una alternativa sensible de adaptación a las diferentes EC preventivas y promocionales, con enfoque multidisciplinario hacia la mejora en la implementación de estrategias dirigidas al desarrollo de competencias profesionales en SM.

\section{Método}

\section{Diseño}

Estudio instrumental que promueve el diseño de herramientas de evaluación e incluso su adaptación (Montero y León, 2002). Psicométrico respecto al establecimiento de modelos de constructo psicológico (Muñiz, 1998). Cuantitativo en el recojo-análisis de datos. Transversal desde el criterio tiempo-espacio (Hernández et al., 2014).

\section{Participantes}

Muestra conformada por 117 estudiantes de la asignatura asociada a «Programas de Intervención en Psicología», durante el primer semestre del año 2021, considerando a 71 alumnos de la Universidad Nacional Federico Villarreal y 46 de la Universidad Privada César Vallejo; 81 mujeres y 36 hombres, todos mayores de 18 años.

\section{Instrumento}

Escala de Aprendizajes Percibidos elaborada para efectos del presente estudio (en función de su contraste psicométrico), inspirado en la composición didáctica y pedagógica del proceso de enseñanza-aprendizaje 
(Rodríguez, 2017). Cuenta con 32 reactivos distribuidos en seis dimensiones: 1) valoración de la EC (7 reactivos), 2) aprendizaje conceptual (5 reactivos), $3)$ aprendizaje procedimental (8 reactivos), 4) aprendizaje actitudinal (4 reactivos), 5) valoración del docente (6 reactivos) y 6) nivel de satisfacción con la EC (2 reactivos), ver Anexo 2; inspirado en la composición didáctica y pedagógica del proceso de enseñanza-aprendizaje (Rodríguez, 2017).

El instrumento cuenta con validez de contenido otorgada por siete catedráticos y/o profesionales expertos en el diseño y ejecución de «Programas de Intervención en Psicología». Este proceso confirma la puntuación $V$ de Aiken total de .96; en relevancia $V=.97$; en representatividad $V=.96 ;$ y en claridad $V=.95$. Asimismo, los 32 reactivos determinan su validez, con un puntaje mínimo de $V=.93$ y máximo de $V=1.00$ (considerando los tres criterios de evaluación por ítem). Ver Anexo 1.

\section{Procedimiento}

Al finalizar el ciclo académico correspondiente a los estudiantes que consintieron participar en el proceso de evaluación de la EC, se aplicó el instrumento por medio de un formulario en línea desde el aplicativo Microsoft Forms, con lo cual se construyó la base de datos.

\section{Análisis de datos}

Los análisis estadísticos se realizaron en el entorno $R$, considerando el Análisis Factorial Confirmatorio (AFC) en tres estimaciones. La estimación con mayor ajuste se evaluó en función de las cargas factoriales por medio del Análisis Factorial de Componentes Principales (AFCP) y según índice de discriminación por factor, para luego determinar el funcionamiento diferencial de ítems respecto de la EC en la universidad pública y en la universidad privada; resultados derivados al análisis de consistencia interna mediante los coeficientes Alfa de Cronbach y Omega de McDonald, para finalizar con el diseño del modelo factorial y sus correlaciones internas. 


\section{Resultados}

Para la evaluación psicométrica de la Escala fueron examinados el modelo de medida y el estructural a partir del análisis factorial confirmatorio, contemplando el contraste entre tres alternativas distintas, a través de estimaciones robustas DWLS con emulación Mplus. La primera consistió en medidas para un modelo de un solo factor; la segunda en el agrupamiento de los ítems para dos factores de segundo orden; y la tercera para 5 factores relacionados. Esta última presentó un mejor ajuste y menor sobresaturación de varianza explicada que los anteriores. Los índices de ajuste obtenidos son presentados en la Tabla 1, donde es apreciable que el modelo confirmatorio de 5 factores posee el menor error.

Tabla 1

Índices de ajuste de los modelos confirmatorios de la Escala

\begin{tabular}{|c|c|c|c|c|c|c|c|c|c|c|}
\hline \multirow{2}{*}{ Modelo } & \multicolumn{4}{|c|}{ Ajuste } & \multicolumn{3}{|c|}{ Comparativos } & \multicolumn{3}{|c|}{ Error } \\
\hline & $\chi^{2}$ & $g l$ & $p$ & GFI & $\mathrm{CFI}$ & TLI & NNFI & RMSEA & SRMR & RMR \\
\hline 5 factores & 130.755 & 454 & 1.000 & .999 & 1.000 & 1.000 & 1.000 & .000 & .065 & .037 \\
\hline Segundo orden & 145.635 & 457 & 1.000 & .999 & 1.000 & 1.000 & 1.000 & .000 & .075 & .046 \\
\hline Unifactorial & 242.652 & 464 & 1.000 & .999 & 1.000 & 1.000 & 1.000 & .000 & .100 & .061 \\
\hline
\end{tabular}

Dadas tales condiciones, se buscó analizar el modelo con pruebas de invarianza paramétrica, obteniéndose homogeneidad entre los parámetros con los criterios de universidad de origen y sexo, sin diferenciales estadísticamente significativos, quedando el modelo con índices de ajuste adecuados $\left(\chi^{2}=130.755, g l=454, p=1.00\right.$, GFI $=.999$, CFI $=1.00$, TLI $=$ $1.00, \mathrm{NNFI}=1.00, \mathrm{RMSEA}=.00, \mathrm{SRMR}=.065, \mathrm{RMR}=.037)$. En tal sentido, en la Tabla 2 se presentan las cargas factoriales para el modelo de 5 factores, para lo cual se asimilaron los ítems 8 y 9 a los factores de aprendizaje actitudinal y procedimental, respectivamente; eliminándose el factor de satisfacción con la experiencia curricular, dado que dichos ítems no convergen para ser cargados en un solo factor. 
Tabla 2

Cargas factoriales del modelo de 5 factores de la Escala

\begin{tabular}{|c|c|c|c|c|c|c|c|c|c|}
\hline \multirow{2}{*}{ Dimensión } & \multirow{2}{*}{ Indicadores por ítem } & \multirow{2}{*}{ Ítem } & \multirow{2}{*}{ E. } & \multirow{2}{*}{ E.E. } & \multirow{2}{*}{$z$} & \multirow{2}{*}{$p$} & \multicolumn{2}{|c|}{ I.C. $(95 \%)$} & \multirow{2}{*}{ Carga } \\
\hline & & & & & & & Inferior & Superior & \\
\hline \multirow{7}{*}{ VEC } & EC y estructura & i1 & 1.000 & .000 & & & 1.000 & 1.000 & .799 \\
\hline & EC y objetivos & i2 & .989 & .069 & 14.294 & $<.001$ & .853 & 1.124 & .801 \\
\hline & $\begin{array}{l}\text { EC como motivación de } \\
\text { desempeño }\end{array}$ & i3 & .813 & .102 & 7.957 & $<.001$ & .613 & 1.013 & .684 \\
\hline & $\begin{array}{l}\text { EC y expectativas de } \\
\text { aprendizaje }\end{array}$ & ¡4 & 1.053 & .102 & 10.369 & $<.001$ & .854 & 1.252 & .826 \\
\hline & EC y valor añadido & ¡5 & .982 & .118 & 8.293 & $<.001$ & .750 & 1.214 & .863 \\
\hline & EC y ejercicio profesional & i6 & .877 & .135 & 6.497 & $<.001$ & .613 & 1.142 & .799 \\
\hline & EC desde la virtualidad & ¡7 & 1.063 & .135 & 7.874 & $<.001$ & .798 & 1.328 & .639 \\
\hline \multirow{6}{*}{ VD } & $\begin{array}{l}\text { Docente y calidad de } \\
\text { ejecución de la EC }\end{array}$ & i10 & 1.000 & .000 & & & 1.000 & 1.000 & .753 \\
\hline & $\begin{array}{l}\text { Docente e interacción } \\
\text { durante la EC }\end{array}$ & i11 & .982 & .091 & 10.813 & $<.001$ & .804 & 1.160 & .872 \\
\hline & $\begin{array}{l}\text { Docente y respuestas } \\
\text { oportunas }\end{array}$ & i12 & 1.040 & .122 & 8.514 & $<.001$ & .801 & 1.280 & .884 \\
\hline & $\begin{array}{l}\text { Docente y recepción de } \\
\text { aportes }\end{array}$ & i13 & .993 & .108 & 9.171 & $<.001$ & .781 & 1.205 & .897 \\
\hline & Docente y actitud alentadora & i14 & .982 & .112 & 8.734 & $<.001$ & .762 & 1.202 & .884 \\
\hline & $\begin{array}{l}\text { Docente como mentor } \\
\text { durante la EC }\end{array}$ & ¡15 & 1.103 & .114 & 9.639 & $<.001$ & .879 & 1.327 & .881 \\
\hline \multirow{5}{*}{ AA } & $\begin{array}{l}\text { EC y participación durante } \\
\text { la EC }\end{array}$ & ¡16 & 1.000 & .000 & & & 1.000 & 1.000 & .577 \\
\hline & $\begin{array}{l}\text { EC y confianza al formular } \\
\text { consultas }\end{array}$ & i17 & 1.317 & .182 & 7.225 & $<.001$ & .960 & 1.674 & .790 \\
\hline & $\begin{array}{l}\text { EC y solicitud de } \\
\text { asesoramiento }\end{array}$ & i18 & 1.356 & .182 & 7.466 & $<.001$ & 1.000 & 1.712 & .814 \\
\hline & $\begin{array}{l}\text { EC y motivación para el } \\
\text { desempeño }\end{array}$ & ¡19 & 1.212 & .194 & 6.249 & $<.001$ & .832 & 1.593 & .779 \\
\hline & $\begin{array}{l}\text { EC y satisfacción con los } \\
\text { aprendizajes }\end{array}$ & i8 & 1.032 & .142 & 7.239 & $<.001$ & .752 & 1.311 & .821 \\
\hline \multirow{5}{*}{$A C$} & $\begin{array}{l}\text { EC y comprensión de } \\
\text { contenidos }\end{array}$ & $\mathrm{i} 20$ & 1.000 & .000 & & & 1.000 & 1.000 & .743 \\
\hline & $\begin{array}{l}\text { EC y asimilación de } \\
\text { objetivos básicos }\end{array}$ & ¡21 & 1.123 & .131 & 8.557 & $<.001$ & .866 & 1.380 & .720 \\
\hline & EC y contenidos prácticos. & i22 & 1.182 & .176 & 6.699 & $<.001$ & .836 & 1.528 & .806 \\
\hline & EC y conceptos relevantes & i23 & .986 & .105 & 9.359 & $<.001$ & .780 & 1.193 & .702 \\
\hline & EC y utilidad de contenidos & i24 & .839 & .196 & 4.279 & $<.001$ & .455 & 1.223 & .742 \\
\hline
\end{tabular}




\begin{tabular}{|c|c|c|c|c|c|c|c|c|c|}
\hline \multirow{2}{*}{ Dimensión } & \multirow{2}{*}{ Indicadores por ítem } & \multirow{2}{*}{ Ítem } & \multirow{2}{*}{ E. } & \multirow{2}{*}{ E.E. } & \multirow{2}{*}{$z$} & \multirow{2}{*}{$p$} & \multicolumn{2}{|c|}{ I.C. $(95 \%)$} & \multirow{2}{*}{ Carga } \\
\hline & & & & & & & Inferior & Superior & \\
\hline \multirow{9}{*}{ AP } & $\begin{array}{l}\text { EC y habilidad para el } \\
\text { diseño de PI }\end{array}$ & i25 & 1.000 & .000 & & & 1.000 & 1.000 & .794 \\
\hline & $\begin{array}{l}\text { EC y elaboración de } \\
\text { matrices de formulación }\end{array}$ & $\mathrm{i} 26$ & .951 & .136 & 7.014 & $<.001$ & .685 & 1.217 & .582 \\
\hline & $\begin{array}{l}\text { EC y elaboración de } \\
\text { matrices de planificación }\end{array}$ & ¡27 & 1.238 & .218 & 5.678 & $<.001$ & .811 & 1.666 & .700 \\
\hline & $\begin{array}{l}\text { EC y elaboración de } \\
\text { sesiones }(\mathrm{PI})\end{array}$ & i28 & .999 & .131 & 7.613 & $<.001$ & .742 & 1.256 & .758 \\
\hline & $\begin{array}{l}\text { EC y estrategias de } \\
\text { facilitación virtual }\end{array}$ & $\mathrm{i} 29$ & 1.090 & .147 & 7.436 & $<.001$ & .803 & 1.377 & .825 \\
\hline & $\begin{array}{l}\text { EC y ejecución de un } \\
\text { programa }\end{array}$ & ¡30 & .806 & .131 & 6.169 & $<.001$ & .550 & 1.063 & .695 \\
\hline & $\begin{array}{l}\text { EC y en monitoreo y } \\
\text { evaluación (PI) }\end{array}$ & ¡31 & 1.136 & .164 & 6.925 & $<.001$ & .814 & 1.457 & .791 \\
\hline & $\begin{array}{l}\text { EC y habilidades } \\
\text { investigativas }\end{array}$ & ¡32 & 1.114 & .161 & 6.905 & $<.001$ & .798 & 1.430 & .750 \\
\hline & $\begin{array}{l}\text { EC y logro del producto } \\
\text { académico }\end{array}$ & i9 & .792 & .179 & 4.420 & $<.001$ & .441 & 1.144 & .610 \\
\hline \multirow{4}{*}{ VEC } & & VD & .313 & .084 & 3.738 & $<.001$ & .149 & .477 & .947 \\
\hline & & $\mathrm{AA}$ & .283 & .080 & 3.520 & $<.001$ & .125 & .440 & .969 \\
\hline & & $A C$ & .255 & .053 & 4.822 & $<.001$ & .152 & .359 & .793 \\
\hline & & AP & .249 & .054 & 4.574 & $<.001$ & .142 & .355 & .757 \\
\hline \multirow{3}{*}{ VD } & & AA & .274 & .087 & 3.169 & .002 & .105 & .444 & .954 \\
\hline & & $A C$ & .231 & .064 & 3.628 & $<.001$ & .106 & .356 & .728 \\
\hline & & $A P$ & .197 & .056 & 3.536 & $<.001$ & .088 & .306 & .608 \\
\hline \multirow{2}{*}{ AA } & & $A C$ & .227 & .053 & 4.320 & $<.001$ & .124 & .330 & .810 \\
\hline & & $A P$ & .205 & .049 & 4.180 & $<.001$ & .109 & .302 & .718 \\
\hline$A C$ & & AP & .283 & .097 & 2.927 & .003 & .093 & .472 & .895 \\
\hline
\end{tabular}

Nota. VEC: Valoración de la EC, VD: Valoración del docente, AA: Aprendizaje actitudinal, AC: Aprendizaje conceptual, AP: Aprendizaje procedimental, E.: Estimación, E.E.: Error estándar, I.C.: Intervalo de confianza.

Se observaron cargas factoriales superiores a .57 en todos los ítems, llegando hasta alrededor de .89 , con covarianzas oscilantes entre .60 y .95 para los factores. En el mismo sentido, las correlaciones ítem-test generadas entre los ítems y los factores correspondientes fueron fuertes, tal como puede apreciarse en la Tabla 3, lo cual pone en evidencia la adecuada capacidad discriminativa de cada ítem por factor. 
Tabla 3

Índices de discriminación por factor

\begin{tabular}{|c|c|c|c|c|c|}
\hline Factor & Ítem & $M$ & $D E$ & $i-j$ & Criterio \\
\hline \multirow{7}{*}{ VEC } & i1 & 4.359 & .725 & .713 & Aceptable \\
\hline & ¡2 & 4.487 & .715 & .813 & Aceptable \\
\hline & ¡3 & 4.547 & .689 & .728 & Aceptable \\
\hline & ¡4 & 4.470 & .738 & .777 & Aceptable \\
\hline & i5 & 4.658 & .659 & .822 & Aceptable \\
\hline & i6 & 4.692 & .636 & .764 & Aceptable \\
\hline & ¡7 & 4.214 & .963 & .567 & Aceptable \\
\hline \multirow{6}{*}{ VD } & i10 & 4.573 & .758 & .747 & Aceptable \\
\hline & i11 & 4.667 & .643 & .843 & Aceptable \\
\hline & ¡12 & 4.590 & .672 & .858 & Aceptable \\
\hline & i13 & 4.658 & .632 & .849 & Aceptable \\
\hline & i14 & 4.650 & .634 & .838 & Aceptable \\
\hline & ¡15 & 4.521 & .714 & .865 & Aceptable \\
\hline \multirow{5}{*}{$A A$} & i8 & 4.581 & .633 & .631 & Aceptable \\
\hline & ¡16 & 4.197 & .873 & .556 & Aceptable \\
\hline & i17 & 4.248 & .840 & .768 & Aceptable \\
\hline & i18 & 4.325 & .839 & .802 & Aceptable \\
\hline & ¡19 & 4.496 & .784 & .670 & Aceptable \\
\hline \multirow{5}{*}{$A C$} & ¡20 & 4.453 & .749 & .726 & Aceptable \\
\hline & i21 & 4.239 & .868 & .769 & Aceptable \\
\hline & i22 & 4.487 & .816 & .762 & Aceptable \\
\hline & i23 & 4.436 & .781 & .781 & Aceptable \\
\hline & i24 & 4.607 & .629 & .542 & Aceptable \\
\hline \multirow{9}{*}{ AP } & i9 & 4.547 & .737 & .419 & Aceptable \\
\hline & i25 & 4.496 & .715 & .717 & Aceptable \\
\hline & i26 & 4.162 & .928 & .603 & Aceptable \\
\hline & i27 & 4.085 & 1.005 & .670 & Aceptable \\
\hline & i28 & 4.556 & .748 & .808 & Aceptable \\
\hline & i29 & 4.521 & .750 & .786 & Aceptable \\
\hline & i30 & 4.658 & .659 & .699 & Aceptable \\
\hline & i31 & 4.462 & .815 & .796 & Aceptable \\
\hline & i32 & 4.410 & .842 & .784 & Aceptable \\
\hline
\end{tabular}

Los resultados del análisis arrojaron correlaciones ítem-test oscilantes entre .56 y .81, siendo superiores al criterio de mínimo valor aceptable de .30 establecido por Guilford. La consistencia interna derivada del análisis 
confirmatorio es mostrada en la Tabla 4, habiendo sido estimada por los coeficientes Omega de McDonald y Alpha, con valores por encima del mínimo aceptable de .70 .

Tabla 4

Medidas de consistencia interna

\begin{tabular}{lccc}
\hline \multicolumn{1}{c}{ Factor } & N $^{\circ}$ de ítems & Alpha & Omega \\
\hline Valoración de la Experiencia Curricular & 7 & .908 & .909 \\
Valoración del docente & 6 & .944 & .944 \\
Aprendizaje actitudinal & 5 & .861 & .863 \\
Aprendizaje conceptual & 5 & .880 & .890 \\
Aprendizaje procedimental & 9 & .909 & .913 \\
\hline
\end{tabular}

Sumándose a tales evidencias, el funcionamiento diferencial de los ítems, tomando como referencia las medias y desviaciones estándar para cada ítem, fueron contrastadas en función de las respuestas de los participantes por el criterio de universidad de origen, reflejando mantenerse homogéneas, como es posible observar en la Figura 1. Es decir, los valores promedio obtenidos para cada ítem por dicho criterio se mantienen dentro de un margen tal que no se reflejan diferencias estadísticamente significativas respecto de las puntuaciones del ítem por el criterio establecido de agrupamiento de las respuestas. Los resultados de este proceso analítico respaldan a los del contraste de invarianza paramétrica referidos en la Tabla 1.

Los coeficientes obtenidos para cada escala se mantuvieron por encima de .86 , llegando hasta .94 , reflejando altas medidas de confiabilidad en las puntuaciones del análisis de la consistencia interna, con los coeficientes vistos.

Finalmente, en la Figura 2 se presenta el diagrama de rutas del modelo de 5 factores, con sus respectivas métricas de variabilidad entre ítems y factores, representándose cocientes de regresión y covarianzas entre ellos. 
Figura 1

Funcionamiento diferencial de los ítems por universidad de origen

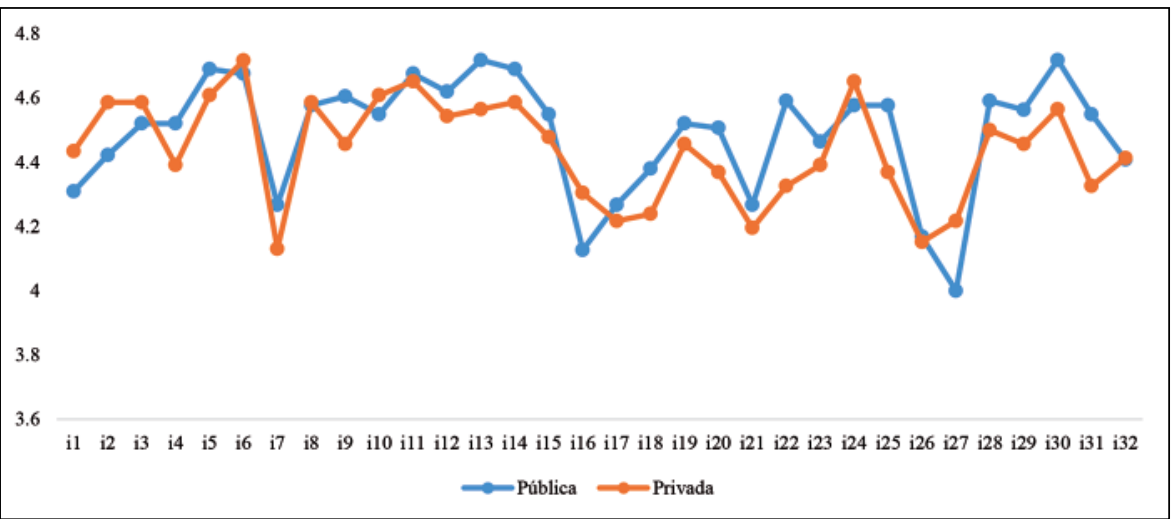

\section{Figura 2}

Modelo confirmatorio de la Escala con 5 factores

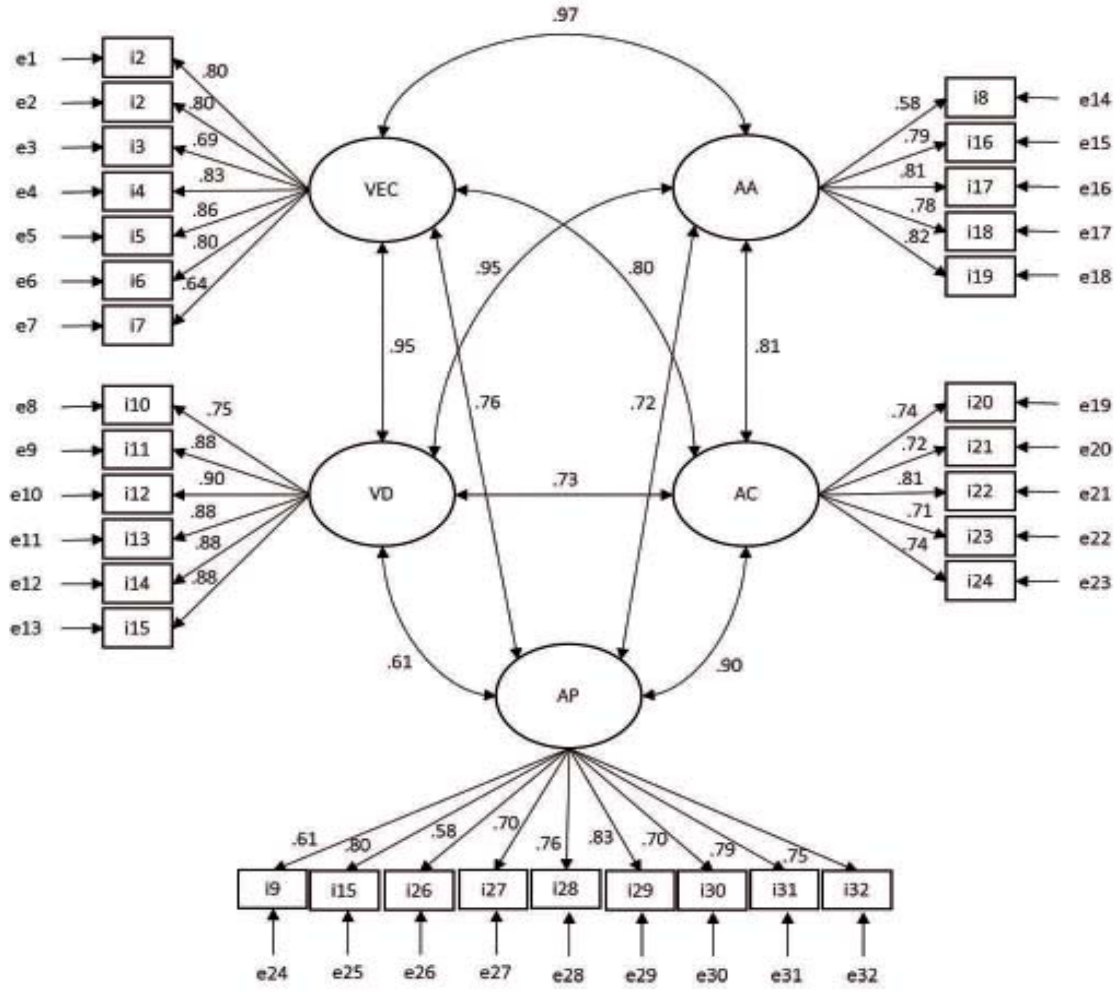




\section{Discusión}

El propósito del estudio se centró en el análisis de las propiedades psicométricas de la Escala de Aprendizajes Percibidos dentro de la EC para un curso de intervención psicológica en estudiantes universitarios de la ciudad de Lima. Las evidencias sostienen la calidad de construcción de la Escala en función de criterios de validez y confiabilidad consistentes, al haberse obtenido del CFA un modelo con 5 factores que se adaptan a criterios de ajuste adecuados (Shi et al., 2019; Cangur y Ercan, 2015). Asimismo, se reflejó consistencia interna en las puntuaciones de los ítems conformando cada factor por encima de .86, lo cual se sumó al análisis como criterio de confiabilidad en las medidas de la Escala, ratificando una vez más el comportamiento multidimensional de las medidas obtenidas con el instrumento (Cangur y Ercan, 2015).

Tales hallazgos comprueban la capacidad del instrumento de posibilitar la replicabilidad del diseño metodológico para el análisis de la EC de estudiantes universitarios respecto a los «Programas de Intervención en Psicología» dentro del ámbito clínico, respondiendo dicha experiencia tanto a la valoración de la materia como a los aprendizajes obtenidos durante el desarrollo del período lectivo, tomando antesala en el desarrollo de instrumentos con bases similares que proporcionen información respecto al proceso (Jeffries, 2005; Arancibia, 2020; Carpio et al., 2021). De este modo, con la construcción de la Escala, en contribución a la formación en SM con base en la investigación formativa, se busca poner en evidencia la calidad de los procesos de aprendizaje de los estudiantes, dando viabilidad al análisis de competencias en el entorno virtual, donde se han visto limitaciones a lo largo de la pandemia y se ha hecho evidente un incremento de problemas como los de depresión y estrés, incluyendo a profesionales de la salud (Quevedo et al., 2020; Huarcaya-Victoria, 2020).

Cabe también mencionar la calidad del comportamiento de las medidas obtenidas con la Escala para discriminar aprendizajes percibidos a nivel de conocimientos, actitudes y procesos, donde los ítems que conforman cada factor constituyen indicadores relevantes de desempeño que podrían tomar equivalencia en procesos metodológicos para el desarrollo de competencias, como en casos de aplicación del aprendizaje basado en proyectos, de la 
enseñanza recíproca o del aprendizaje basado en problemas, con evidencias complementarias de desempeño (Carrasco et al., 2015; Espada et al., 2021; Gil-Galván, 2018).

Se evidencia la importancia de continuar revisando las propiedades de la Escala en otros profesionales de la salud, posibilitando el análisis del comportamiento diferencial de los ítems más allá del criterio de tipo de gestión universitaria (pública y privada), considerando aspectos como los de carreras universitarias conexas, condiciones laborales o de ejercicio de la profesión y antecedentes asociados a la calidad de los procesos de aprendizaje, con factores de riesgo potencialmente intervinientes como problemas de sueño y vigilia, alimentación, exposición a enfermedades y diagnósticos de estrés, ansiedad o depresión como desencadenantes de deterioro de la salud mental (Huarcaya-Victoria, 2020). Considerar la variabilidad de condiciones en torno a tales términos podría incrementar la posibilidad de generalizar las medidas de la Escala con parámetros de invarianza que definan a la EC en la métrica correspondiente, explicada por sí misma como constructo psicológico con un modelo sólido para el análisis de las evidencias en la formación profesional (Shi et al., 2019; Lara, 2014; Morales, 2012; Oliden, 2003).

Los roles del estudiante, en función del compromiso social derivado de la formación en SM, se convierten también en un punto de análisis de la competencia esperada, donde resulta recomendable verificar dicho aspecto en contraste con los indicadores de la Escala, dado el énfasis en carreras de servicio a partir de las cuales se concibe esta condición como inherente al desempeño de los estudiantes y mentores (Rosario et al., 2021). Las posibilidades de análisis sobre este propósito en multinivel podrían proporcionar complementariedad a las características del desempeño docente en procesos formativos universitarios para tales líneas de carrera, con miras hacia un impacto social sostenible en poblaciones beneficiarias de los servicios. 


\section{Conclusiones}

La Escala de Aprendizajes Percibidos de 32 ítems reformuló su estructura a cinco dimensiones, con la distribución de los dos reactivos asociados con «Satisfacción con la EC», en los rubros actitudinal y conceptual. Modelo dimensional que contiene buena calidad de ajuste y menor sobresaturación de varianza explicada.

Las cargas factoriales de los reactivos y las covarianzas dimensionales son adecuadas, y presentan un análisis ítem-test que garantiza la aceptabilidad de cada reactivo según dimensión.

Los reactivos poseen un comportamiento estable en el contraste de las poblaciones evaluadas, denotando confiabilidad y consistencia interna; asimismo, mantienen confiabilidad dimensional según los coeficientes alfa y omega.

El nuevo modelo confirma correlaciones altamente significativas entre la tríada perceptiva: valoración docente-aprendizaje actitudinal-contenidos de la EC; incluyéndose la correlación alta entre aprendizajes conceptuales y aprendizajes procedimentales (como es predecible), así como una tendencia considerable hacia lo actitudinal y la valoración de contenidos.

Finalmente, las evidencias psicométricas de la Escala de Aprendizajes Percibidos en la EC «Programas de Intervención en Psicología» presentan un instrumento, válido, confiable, con una estructura factorial que sustenta un constructo explicativo del aprendizaje desde los paradigmas didáctico y pedagógico, susceptible de adaptación a otras EC asociadas a programas de intervención de índole multidisciplinaria. 


\section{Referencias}

Arancibia, A., Paredes, P., Salibe, S. y Alfaro, M. (2020). Propiedades psicométricas de la versión en español de un cuestionario para evaluar la simulación clínica en titulaciones de Ciencias de la Salud. Enfermería Clínica. https://doi.org/10.1016/j.enfcli.2020.08.003

Cangur, S. y Ercan, I. (2015). Comparison of model fit indices used in structural equation modeling under multivariate normality. Journal of Modern Applied Statistical Methods, 14(1), 152-167. https://digitalcommons.wayne.edu/ cgi/viewcontent.cgi?referer=https://scholar.google.com/\&httpsredir=1\&article=1810\&context=jmasm

Carpio, H. del, Carpio, P. A. del, Carpio, S. R. B. del y García-Peñalvo, F. J. (2021). Validez de instrumento: percepción del aprendizaje virtual durante la COVID-19. Espacios, 38(60), 111-125. https://www.scopus.com/ record/display.uri?eid=2-s2.0-85038615072\&origin=resultslist

Carrasco, A., Donoso, J. A., Duarte-Atoche, T., Hernández, J. L. y López, R. (2015). Diseño y validación de un cuestionario que mide la percepción de efectividad del uso de metodologías de participación activa (CEMPA). El caso del Aprendizaje Basado en Proyectos (ABPrj) en la docencia de la contabilidad. Innovar, 25(58), 143158. https://doi.org/10.15446/innovar.v25n58.52439

Echeverría-Ramírez, J. A. y Mazzitelli, C. (2021). Estudio de la percepción sobre los factores institucionales que influyen en el rendimiento académico de estudiantes de la Universidad Estatal a Distancia de Costa Rica. Revista Electrónica Educare, 25(2), 1-20. https://doi.org/10.15359/ree.25-2.18

Espada, M., Fernández, M. y Calero, J. C. (2021). Validación española del cuestionario experiencia y percepción de los estudiantes del espectro de estilos de enseñanza en Educación Física. Journal of Sport and Health Research, 13(2), 305-308. https://recyt.fecyt.es/index.php/JSHR/article/view/89607

Gil-Galván, R. (2018). El uso del aprendizaje basado en problemas en la enseñanza universitaria. Análisis de las competencias adquiridas y su impacto. Revista Mexicana de Investigación Educativa, 23(76), 73-93. http:// uww.scielo.org.mx/pdf/rmie/v23n76/1405-6666-rmie-23-76-73.pdf

Hernández, R., Fernández, C. y Baptista, P. (2014). Metodología de la investigación. Sexta edición. McGraw Hill. https://www.uca.ac.cr/wp-content/uploads/2017/10/Investigacion.pdf

Huarcaya-Victoria, J. (2020). Consideraciones sobre la salud mental en la pandemia de COVID-19. Revista Peruana de Medicina Experimental y Salud Pública, 37(2), 327-334. http://dx.doi.org/10.17843/ rpmesp.2020.372.5419

Jeffries, P. R. (2005). A framework for designing, implementing, and evaluating: Simulations used as teaching strategies in nursing. Nurs Educ Perspect, 26(2), 96-103. https://pubmed.ncbi.nlm.nih.gov/15921126/

Lara, A. (2014). Introducción a las ecuaciones estructurales en AMOS y R. Memoria de Curso de Máster en Estadística. Universidad de Granada. https://masteres.ugr.es/moea/pages/curso201314/tfm1314/tfmseptiembre1314/memoriamasterlarahormigoantonio/

León, X. M., Mendoza, M. M. y Gilar, R. (2021). Clima de aula y rendimiento académico: apuntes en torno al contexto universitario. Revista Venezolana de Gerencia, 26(5), 140-156. https://doi.org/10.52080/rvgluz.26.e5.10

Montero, I. y León, O. (2002). Clasificación de metodologías de investigación en psicología. Revista Internacional de Psicología Clínica y de la Salud, 2(3), 503-508. http://aepc.es/ijchp/articulos_pdf/ijchp-53.pdf

Morales, P. (2012). Análisis de ítems en las pruebas objetivas. Universidad Pontificia Comillas.

Muñiz, J. (1998). Medición de lo psicológico. Psicothema, 10(1), 1-21. http://www.psicothema.es/pdf/138.pdf

Oliden, P. E. (2003). Sobre la validez de los tests. Psicothema, 15(2), 315-321. https://www.redalyc.org/pdf/727/ 72715225.pdf 
Quevedo, N. V., García, N., Cañizares, F. P. y Gavilánez, S. M. (2020). La formación del conocimiento investigativo conceptual, actitudinal y procedimental en estudiantes universitarios. Conrado, 16(75), 364-371. http://scielo.sld.cu/ pdf/rc/v16n75/1990-8644-rc-16-75-364.pdf

Rodríguez, E. M. R. (2017). Competencias didácticas-pedagógicas del docente en la transformación del estudiante universitario. Orbis: Revista de Ciencias Humanas, 13(37), 41-55. https://dialnet.unirioja.es/servlet/articulo? codigo $=7158994$

Rojas-Bolívar, D., Bardalez-García, B., Bravo-Vásquez, M. L., Arroyo-Ramírez, F. A. y Yon-Leau, C. (2021). Percepción del ambiente educacional y rendimiento académico en una escuela de medicina de Lima: un estudio longitudinal. Educación Médica, 22(5), 409-413. https://doi.org/10.1016/j.edumed.2020.11.009

Romero-Rodríguez, J. M., Díaz, I. A., Hinojo-Lucena, F. J. y Gómez-García, G. (2021). Uso de los dispositivos móviles en educación superior: relación con el rendimiento académico y la autorregulación del aprendizaje. Revista Complutense de Educación, 32(3), 327-335. https://doi.org/10.5209/rced.70180

Rosario, E. A., Medina, A. R. y Sánchez, J. I. (2021). Challenges of university health education before COVID19. [Carta al editor]. Educación Médica, 22(1), S30. https://doi.org/10.1016/j.edumed.2020.09.007

Shi, D., Lee, T. y Maydeu-Olivares, A. (2019). Comprensión del efecto del tamaño del modelo en los índices de ajuste SEM. Medición educativa y psicológica, 79(2), 310-334. https://doi.org/10.1177/0013164418783530 


\section{Anexo 1}

Validez de contenido de la Escala de Aprendizajes Percibidos

\begin{tabular}{|c|c|c|c|c|c|c|c|c|c|c|c|c|c|}
\hline Ítem & Criterio & 舀 & $\begin{array}{l}N \\
N \\
\tilde{N}\end{array}$ & $\stackrel{m}{\mathscr{N}}$ & 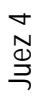 & $\begin{array}{l}n \\
\mathbb{N} \\
\stackrel{n}{n}\end{array}$ & $\begin{array}{l}0 \\
N \\
\stackrel{N}{n}\end{array}$ & $\stackrel{N}{N}$ & Media & $D E$ & $\begin{array}{l}V \text { de Aiken } \\
\text { por criterio }\end{array}$ & $\begin{array}{l}V \text { de Aiken } \\
\text { por ítem }\end{array}$ & $\begin{array}{c}\text { Interpretación } \\
V \text { de Aiken }\end{array}$ \\
\hline \multirow{3}{*}{1} & Relevancia & 3 & 3 & 2 & 3 & 3 & 3 & 3 & 2.86 & .38 & .95 & \multirow{3}{*}{.95} & \multirow{3}{*}{ Válida } \\
\hline & Representatividad & 3 & 3 & 3 & 3 & 3 & 3 & 2 & 2.86 & .38 & .95 & & \\
\hline & Claridad & 3 & 3 & 3 & 3 & 3 & 3 & 2 & 2.86 & .38 & .95 & & \\
\hline \multirow{3}{*}{2} & Relevancia & 3 & 3 & 2 & 3 & 3 & 3 & 3 & 2.86 & .38 & .95 & \multirow{3}{*}{.93} & \multirow{3}{*}{ Válida } \\
\hline & Representatividad & 3 & 2 & 2 & 3 & 3 & 3 & 3 & 2.71 & .49 & .9 & & \\
\hline & Claridad & 3 & 2 & 3 & 3 & 3 & 3 & 3 & 2.86 & .38 & .95 & & \\
\hline \multirow{3}{*}{3} & Relevancia & 3 & 3 & 3 & 3 & 3 & 3 & 2 & 2.86 & .38 & .95 & \multirow{3}{*}{.95} & \multirow{3}{*}{ Válida } \\
\hline & Representatividad & 3 & 3 & 3 & 3 & 3 & 3 & 2 & 2.86 & .38 & .95 & & \\
\hline & Claridad & 3 & 3 & 3 & 3 & 3 & 3 & 2 & 2.86 & .38 & .95 & & \\
\hline \multirow{3}{*}{4} & Relevancia & 3 & 3 & 3 & 3 & 3 & 3 & 3 & 3 & 0 & 1 & \multirow{3}{*}{.94} & \multirow{3}{*}{ Válida } \\
\hline & Representatividad & 3 & 2 & 3 & 3 & 3 & 3 & 3 & 2.86 & .38 & .95 & & \\
\hline & Claridad & 2 & 2 & 3 & 3 & 3 & 2 & 3 & 2.57 & .53 & .86 & & \\
\hline \multirow{3}{*}{5} & Relevancia & 3 & 3 & 2 & 3 & 3 & 3 & 3 & 2.86 & .38 & .95 & \multirow{3}{*}{.96} & \multirow{3}{*}{ Válida } \\
\hline & Representatividad & 3 & 3 & 3 & 3 & 3 & 3 & 3 & 3 & 0 & 1 & & \\
\hline & Claridad & 3 & 2 & 3 & 3 & 3 & 3 & 3 & 2.86 & .38 & .95 & & \\
\hline \multirow{3}{*}{6} & Relevancia & 3 & 3 & 2 & 3 & 3 & 3 & 3 & 2.86 & .38 & .95 & \multirow{3}{*}{.95} & \multirow{3}{*}{ Válida } \\
\hline & Representatividad & 3 & 3 & 2 & 3 & 3 & 3 & 3 & 2.86 & .38 & .95 & & \\
\hline & Claridad & 3 & 3 & 2 & 3 & 3 & 3 & 3 & 2.86 & .38 & .95 & & \\
\hline \multirow{3}{*}{7} & Relevancia & 3 & 3 & 2 & 3 & 3 & 3 & 3 & 2.86 & .38 & .95 & \multirow{3}{*}{.93} & \multirow{3}{*}{ Válida } \\
\hline & Representatividad & 3 & 2 & 2 & 3 & 3 & 3 & 3 & 2.71 & .49 & .9 & & \\
\hline & Claridad & 3 & 3 & 2 & 3 & 3 & 3 & 3 & 2.86 & .38 & .95 & & \\
\hline \multirow{3}{*}{8} & Relevancia & 3 & 3 & 3 & 3 & 3 & 3 & 3 & 3 & 0 & 1 & \multirow{3}{*}{.95} & \multirow{3}{*}{ Válida } \\
\hline & Representatividad & 3 & 2 & 3 & 3 & 3 & 3 & 3 & 2.86 & .38 & .95 & & \\
\hline & Claridad & 2 & 2 & 3 & 3 & 3 & 3 & 3 & 2.71 & .49 & .9 & & \\
\hline \multirow{3}{*}{9} & Relevancia & 3 & 2 & 3 & 3 & 3 & 3 & 3 & 2.86 & .38 & .95 & \multirow{3}{*}{.93} & \multirow{3}{*}{ Válida } \\
\hline & Representatividad & 3 & 2 & 3 & 3 & 3 & 3 & 3 & 2.86 & .38 & .95 & & \\
\hline & Claridad & 2 & 2 & 3 & 3 & 3 & 3 & 3 & 2.71 & .49 & .9 & & \\
\hline & Relevancia & 3 & 3 & 3 & 3 & 3 & 3 & 3 & 3 & 0 & 1 & & \\
\hline 10 & Representatividad & 3 & 3 & 3 & 3 & 3 & 3 & 3 & 3 & 0 & 1 & .98 & Válida \\
\hline & Claridad & 3 & 3 & 3 & 2 & 3 & 3 & 3 & 2.86 & .38 & .95 & & \\
\hline
\end{tabular}




\begin{tabular}{|c|c|c|c|c|c|c|c|c|c|c|c|c|c|}
\hline Ítem & Criterio & 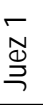 & $\stackrel{N}{N}$ & $\begin{array}{l}m \\
\stackrel{N}{2} \\
\tilde{n}\end{array}$ & $\begin{array}{l}\stackrel{\nabla}{2} \\
\stackrel{\otimes}{\partial}\end{array}$ & $\begin{array}{l}10 \\
\mathbb{N} \\
\stackrel{n}{n}\end{array}$ & $\begin{array}{l}0 \\
N \\
\stackrel{N}{n}\end{array}$ & $\stackrel{N}{\stackrel{N}{n}}$ & Media & $D E$ & $\begin{array}{l}V \text { de Aiken } \\
\text { por criterio }\end{array}$ & $\begin{array}{c}V \text { de Aiken } \\
\text { por ítem }\end{array}$ & $\begin{array}{c}\text { Interpretación } \\
V \text { de Aiken }\end{array}$ \\
\hline \multirow{3}{*}{11} & Relevancia & 3 & 2 & 3 & 3 & 3 & 3 & 3 & 2.86 & .38 & .95 & \multirow{3}{*}{.96} & \multirow{3}{*}{ Válida } \\
\hline & Representatividad & 3 & 3 & 3 & 3 & 3 & 3 & 3 & 3 & 0 & 1 & & \\
\hline & Claridad & 3 & 2 & 3 & 3 & 3 & 3 & 3 & 2.86 & .38 & .95 & & \\
\hline \multirow{3}{*}{12} & Relevancia & 3 & 3 & 3 & 3 & 3 & 3 & 3 & 3 & 0 & 1 & \multirow{3}{*}{.98} & \multirow{3}{*}{ Válida } \\
\hline & Representatividad & 3 & 3 & 2 & 3 & 3 & 3 & 3 & 2.86 & .38 & .95 & & \\
\hline & Claridad & 3 & 3 & 3 & 3 & 3 & 3 & 3 & 3 & 0 & 1 & & \\
\hline \multirow{3}{*}{13} & Relevancia & 3 & 3 & 3 & 3 & 3 & 3 & 3 & 3 & 0 & 1 & \multirow{3}{*}{.98} & \multirow{3}{*}{ Válida } \\
\hline & Representatividad & 3 & 3 & 3 & 3 & 3 & 3 & 3 & 3 & 0 & 1 & & \\
\hline & Claridad & 3 & 3 & 3 & 2 & 3 & 3 & 3 & 2.86 & .38 & .95 & & \\
\hline \multirow{3}{*}{14} & Relevancia & 3 & 2 & 3 & 3 & 3 & 3 & 3 & 2.86 & .38 & .95 & \multirow{3}{*}{.95} & \multirow{3}{*}{ Válida } \\
\hline & Representatividad & 3 & 3 & 3 & 3 & 3 & 3 & 3 & 3 & 0 & 1 & & \\
\hline & Claridad & 3 & 2 & 3 & 3 & 3 & 2 & 3 & 2.71 & .49 & .9 & & \\
\hline \multirow{3}{*}{15} & Relevancia & 3 & 3 & 3 & 3 & 3 & 3 & 3 & 3 & 0 & 1 & \multirow{3}{*}{.96} & \multirow{3}{*}{ Válida } \\
\hline & Representatividad & 3 & 3 & 2 & 3 & 3 & 2 & 3 & 2.71 & .49 & .9 & & \\
\hline & Claridad & 3 & 3 & 3 & 3 & 3 & 3 & 3 & 3 & 0 & 1 & & \\
\hline \multirow{3}{*}{16} & Relevancia & 3 & 3 & 3 & 3 & 3 & 3 & 3 & 3 & 0 & 1 & \multirow{3}{*}{1} & \multirow{3}{*}{ Válida } \\
\hline & Representatividad & 3 & 3 & 3 & 3 & 3 & 3 & 3 & 3 & 0 & 1 & & \\
\hline & Claridad & 3 & 3 & 3 & 3 & 3 & 3 & 3 & 3 & 0 & 1 & & \\
\hline \multirow{3}{*}{17} & Relevancia & 3 & 2 & 3 & 3 & 3 & 3 & 2 & 2.71 & .49 & .9 & \multirow{3}{*}{.91} & \multirow{3}{*}{ Válida } \\
\hline & Representatividad & 3 & 3 & 3 & 3 & 3 & 3 & 2 & 2.86 & .38 & .95 & & \\
\hline & Claridad & 3 & 2 & 3 & 3 & 3 & 3 & 2 & 2.71 & .49 & .9 & & \\
\hline \multirow{3}{*}{18} & Relevancia & 3 & 3 & 3 & 3 & 3 & 3 & 2 & 2.86 & .38 & .95 & \multirow{3}{*}{.93} & \multirow{3}{*}{ Válida } \\
\hline & Representatividad & 3 & 3 & 2 & 3 & 3 & 3 & 2 & 2.71 & .49 & .9 & & \\
\hline & Claridad & 3 & 3 & 3 & 3 & 3 & 3 & 2 & 2.86 & .38 & .95 & & \\
\hline \multirow{3}{*}{19} & Relevancia & 3 & 3 & 3 & 3 & 3 & 3 & 3 & 3 & 0 & 1 & & \\
\hline & Representatividad & 3 & 3 & 3 & 3 & 3 & 3 & 3 & 3 & 0 & 1 & 1 & Válida \\
\hline & Claridad & 3 & 3 & 3 & 3 & 3 & 3 & 3 & 3 & 0 & 1 & & \\
\hline & Relevancia & 3 & 2 & 3 & 3 & 3 & 3 & 3 & 2.86 & .38 & .95 & & \\
\hline 20 & Representatividad & 3 & 3 & 3 & 3 & 3 & 3 & 3 & 3 & 0 & 1 & .96 & Válida \\
\hline & Claridad & 3 & 2 & 3 & 3 & 3 & 3 & 3 & 2.86 & .38 & .95 & & \\
\hline & Relevancia & 3 & 3 & 3 & 3 & 3 & 3 & 3 & 3 & 0 & 1 & & \\
\hline 21 & Representatividad & 3 & 3 & 2 & 3 & 3 & 3 & 3 & 2.86 & .38 & .95 & .98 & Válida \\
\hline & Claridad & 3 & 3 & 3 & 3 & 3 & 3 & 3 & 3 & 0 & 1 & & \\
\hline
\end{tabular}




\begin{tabular}{|c|c|c|c|c|c|c|c|c|c|c|c|c|c|}
\hline Ítem & Criterio & $\begin{array}{l}-1 \\
\stackrel{N}{n}\end{array}$ & $\begin{array}{l}N \\
N \\
\stackrel{N}{n}\end{array}$ & $\begin{array}{l}m \\
\stackrel{N}{2}\end{array}$ & $\begin{array}{l}\stackrel{\nabla}{N} \\
\stackrel{N}{\nu}\end{array}$ & $\begin{array}{l}n \\
\stackrel{N}{N} \\
\stackrel{n}{n}\end{array}$ & $\begin{array}{l}0 \\
N \\
\stackrel{Q}{n}\end{array}$ & $\begin{array}{l}\hat{N} \\
\stackrel{Q}{n}\end{array}$ & Media & $D E$ & $\begin{array}{l}V \text { de Aiken } \\
\text { por criterio }\end{array}$ & $\begin{array}{c}V \text { de Aiken } \\
\text { por ítem }\end{array}$ & $\begin{array}{c}\text { Interpretación } \\
V \text { de Aiken }\end{array}$ \\
\hline \multirow{3}{*}{22} & Relevancia & 3 & 3 & 3 & 3 & 3 & 3 & 3 & 3 & 0 & 1 & \multirow{3}{*}{.96} & \multirow{3}{*}{ Válida } \\
\hline & Representatividad & 3 & 3 & 2 & 3 & 3 & 3 & 3 & 2.86 & .38 & .95 & & \\
\hline & Claridad & 3 & 3 & 3 & 3 & 3 & 2 & 3 & 2.86 & .38 & .95 & & \\
\hline \multirow{3}{*}{23} & Relevancia & 3 & 3 & 3 & 3 & 3 & 3 & 3 & 3 & 0 & 1 & \multirow{3}{*}{1} & \multirow{3}{*}{ Válida } \\
\hline & Representatividad & 3 & 3 & 3 & 3 & 3 & 3 & 3 & 3 & 0 & 1 & & \\
\hline & Claridad & 3 & 3 & 3 & 3 & 3 & 3 & 3 & 3 & 0 & 1 & & \\
\hline \multirow{3}{*}{24} & Relevancia & 3 & 2 & 3 & 3 & 3 & 3 & 3 & 2.86 & .38 & .95 & \multirow{3}{*}{.95} & \multirow{3}{*}{ Válida } \\
\hline & Representatividad & 3 & 3 & 3 & 3 & 3 & 3 & 3 & 3 & 0 & 1 & & \\
\hline & Claridad & 3 & 2 & 3 & 3 & 3 & 2 & 3 & 2.71 & .49 & .9 & & \\
\hline \multirow{3}{*}{25} & Relevancia & 3 & 3 & 3 & 3 & 3 & 3 & 3 & 3 & 0 & 1 & \multirow{3}{*}{.96} & \multirow{3}{*}{ Válida } \\
\hline & Representatividad & 3 & 3 & 2 & 3 & 3 & 3 & 3 & 2.86 & .38 & .95 & & \\
\hline & Claridad & 3 & 3 & 3 & 3 & 3 & 2 & 3 & 2.86 & .38 & .95 & & \\
\hline \multirow{3}{*}{26} & Relevancia & 3 & 3 & 3 & 3 & 3 & 3 & 3 & 3 & 0 & 1 & \multirow{3}{*}{1} & \multirow{3}{*}{ Válida } \\
\hline & Representatividad & 3 & 3 & 3 & 3 & 3 & 3 & 3 & 3 & 0 & 1 & & \\
\hline & Claridad & 3 & 3 & 3 & 3 & 3 & 3 & 3 & 3 & 0 & 1 & & \\
\hline \multirow{3}{*}{27} & Relevancia & 3 & 2 & 3 & 3 & 3 & 3 & 3 & 2.86 & .38 & .95 & \multirow{3}{*}{.95} & \multirow{3}{*}{ Válida } \\
\hline & Representatividad & 3 & 3 & 3 & 3 & 3 & 3 & 3 & 3 & 0 & 1 & & \\
\hline & Claridad & 3 & 2 & 3 & 3 & 3 & 2 & 3 & 2.71 & .49 & .9 & & \\
\hline \multirow{3}{*}{28} & Relevancia & 3 & 3 & 3 & 3 & 3 & 3 & 3 & 3 & 0 & 1 & \multirow{3}{*}{.96} & \multirow{3}{*}{ Válida } \\
\hline & Representatividad & 3 & 3 & 2 & 3 & 3 & 3 & 3 & 2.86 & .38 & .95 & & \\
\hline & Claridad & 3 & 3 & 3 & 3 & 3 & 2 & 3 & 2.86 & .38 & .95 & & \\
\hline \multirow{3}{*}{29} & Relevancia & 3 & 3 & 3 & 3 & 3 & 3 & 3 & 3 & 0 & 1 & \multirow{3}{*}{1} & \multirow{3}{*}{ Válida } \\
\hline & Representatividad & 3 & 3 & 3 & 3 & 3 & 3 & 3 & 3 & 0 & 1 & & \\
\hline & Claridad & 3 & 3 & 3 & 3 & 3 & 3 & 3 & 3 & 0 & 1 & & \\
\hline \multirow{3}{*}{30} & Relevancia & 3 & 2 & 3 & 3 & 3 & 3 & 3 & 2.86 & .38 & .95 & & \\
\hline & Representatividad & 3 & 3 & 3 & 3 & 3 & 3 & 3 & 3 & 0 & 1 & .95 & Válida \\
\hline & Claridad & 3 & 2 & 3 & 3 & 3 & 2 & 3 & 2.71 & .49 & .9 & & \\
\hline & Relevancia & 3 & 3 & 3 & 3 & 3 & 3 & 3 & 3 & 0 & 1 & & \\
\hline 31 & Representatividad & 3 & 3 & 2 & 3 & 3 & 3 & 3 & 2.86 & .38 & .95 & .96 & Válida \\
\hline & Claridad & 3 & 3 & 3 & 3 & 3 & 2 & 3 & 2.86 & .38 & .95 & & \\
\hline & Relevancia & 3 & 3 & 3 & 3 & 3 & 3 & 3 & 3 & 0 & 1 & & \\
\hline 32 & Representatividad & 3 & 3 & 2 & 3 & 3 & 3 & 3 & 2.86 & .38 & .95 & .96 & Válida \\
\hline & Claridad & 3 & 3 & 3 & 3 & 3 & 2 & 3 & 2.86 & .38 & .95 & & \\
\hline
\end{tabular}




\section{Anexo 2 \\ Escala de Aprendizajes Percibidos en la Experiencia Curricular «Programas de Intervención en Psicología» - Versión de 5 factores}

\begin{tabular}{lllllll}
\hline \multirow{2}{*}{ Factor $\mathrm{N}^{\circ}$ Or } & Ítems & \multicolumn{4}{c}{ Nivel } \\
\cline { 3 - 6 } & & 1 & 2 & 3 & 4 & 5 \\
\hline
\end{tabular}

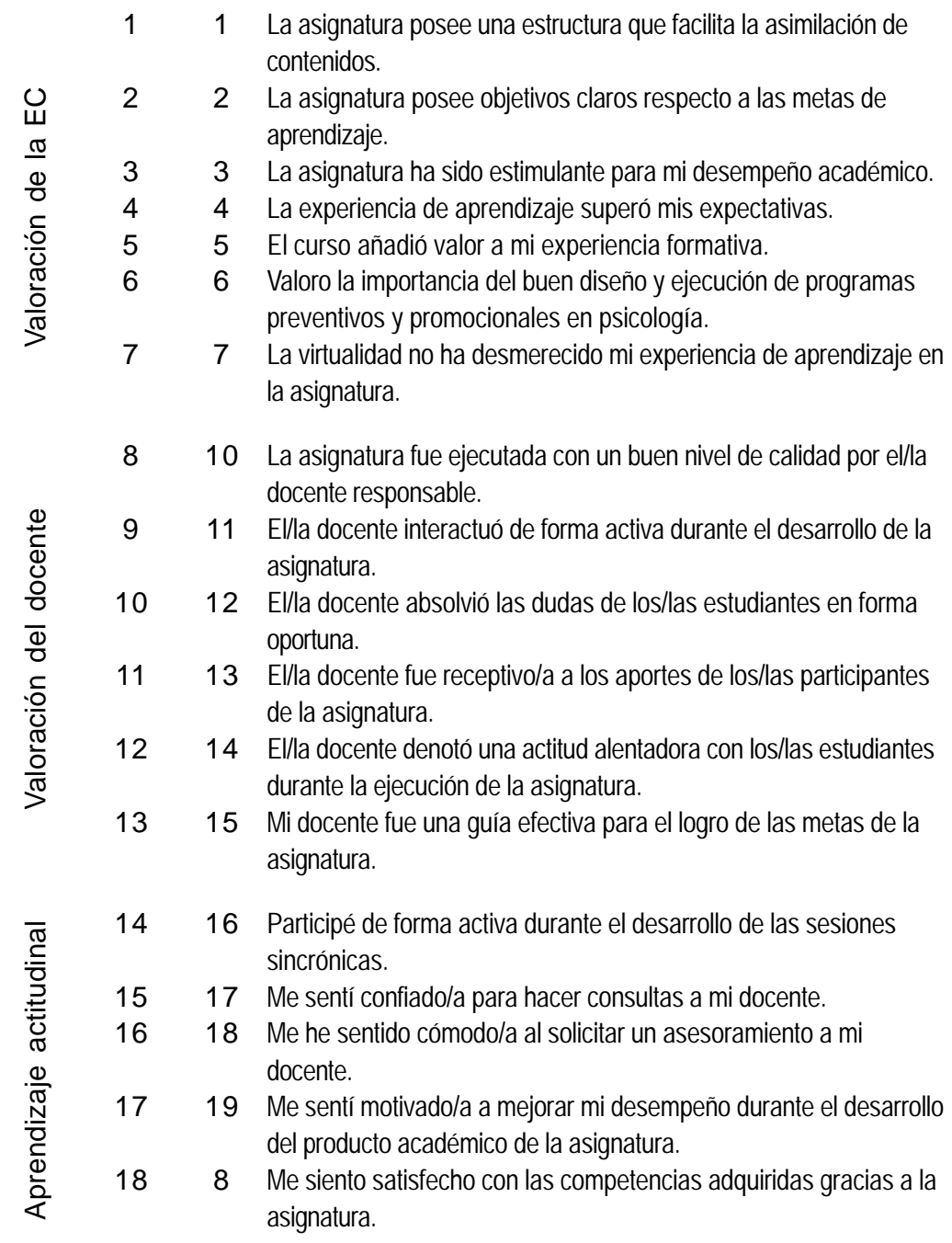




\begin{tabular}{lllllll}
\hline Factor $\mathrm{N}^{\circ}$ Or & Ítems & \multicolumn{4}{c}{ Nivel } \\
\cline { 3 - 6 } & & 1 & 2 & 3 & 4 & 5 \\
\hline
\end{tabular}

1920 Adquirí adecuada comprensión de los contenidos básicos del curso.

$\stackrel{\frac{0}{\sigma}}{\frac{\pi}{C}} 2021$ Tengo la capacidad de describir con claridad los objetivos básicos del curso.

2122 Aprendí gran cantidad de contenidos prácticos.

2223 Identifico los conceptos más importantes del curso.

2324 El contenido de la asignatura fue de utilidad respecto a mi futuro ejercicio profesional.

2425 He mejorado mis habilidades para el diseño y formulación de programas preventivos.
- 2526 Poseo habilidad para la elaboración de matrices de la fase de : formulación (Árbol de problemas y/o espina de Ishikawa).
Фै $26 \quad 27$ Aprendí a elaborar instrumentos para la fase de planificación de programas (Matriz de Marco Lógico).
¿ 2728 He incrementado mi capacidad para la elaboración de sesiones de
a aprendizaje efectivas.
$\stackrel{\frac{0}{\sigma}}{\sigma} 28 \quad 29$ He incorporado nuevas estrategias de facilitación de sesiones de aprendizaje preventivo y/o promocional en psicología, desde la virtualidad.
2930 Logré ejecutar el programa que diseñé.
3031 Utilizo estrategias para el monitoreo y evaluación de programas.
3132 Puse en práctica conocimientos básicos en investigación para la evaluación de los programas ejecutados.

329 Me siento satisfecho con los logros de mi producto académico (programa diseñado y ejecutado). 


\begin{abstract}
Anexo 3
Escala de Aprendizajes Percibidos en la Experiencia Curricular «Programas de Intervención en Psicología»

Por: Marivel Aguirre-Morales y Lizley Tantaleán-Terrones
\end{abstract}

En el presente cuestionario se evalúa la experiencia de aprendizaje de los estudiantes respecto a la asignatura « ", implementada de forma virtual en la Facultad/ Escuela de Psicología de la Universidad , durante el ciclo académico

Como docentes valoramos la importancia de conocer su percepción y apreciación de aprendizajes, como un elemento valioso para la mejora continua de la asignatura como experiencia formativa.

Asimismo, se garantiza la confidencialidad de sus respuestas, cuyo conocimiento es de exclusivo interés académico y de investigación formativa.

Finalmente, se indica que al contestar el presente cuestionario usted expresa su conformidad en participar del proceso de evaluación de la asignatura.

Lea con detenimiento las preguntas y evalúe su experiencia respecto a la asignatura « ".

$\mathrm{Su}$ respuesta será indicada en cinco niveles: el nivel «Uno $=1$ » implica la más baja consideración o apreciación, y el nivel «Cinco $=5$ » la más elevada consideración o apreciación. 


$\mathrm{N}^{\circ} \quad$ Ítems $\quad$\begin{tabular}{c} 
Nivel \\
\cline { 2 - 4 } \\
\cline { 1 - 5 }
\end{tabular}

1 La asignatura posee una estructura que facilita la asimilación de contenidos.

2 La asignatura posee objetivos claros respecto a las metas de aprendizaje.

3 La asignatura ha sido estimulante para mi desempeño académico.

4 La experiencia de aprendizaje superó mis expectativas.

5 El curso añadió valor a mi experiencia formativa.

6 Valoro la importancia del buen diseño y ejecución de programas preventivos y promocionales en psicología.

7 La virtualidad no ha desmerecido mi experiencia de aprendizaje en la asignatura.

8 La asignatura fue ejecutada con un buen nivel de calidad por el/la docente responsable.

$9 \mathrm{El} / \mathrm{la}$ docente interactuó de forma activa durante el desarrollo de la asignatura.

$10 \mathrm{El} /$ la docente absolvió las dudas de los/las estudiantes en forma oportuna.

$11 \mathrm{El} / \mathrm{la}$ docente fue receptivo/a con los aportes de los/las participantes de la asignatura.

12 El/la docente denotó una actitud alentadora con los/las estudiantes durante la ejecución de la asignatura.

13 Mi docente fue una guía efectiva para el logro de las metas de la asignatura.

14 Participé de forma activa durante el desarrollo de las sesiones sincrónicas.

15 Me sentí confiado/a para hacer consultas a mi docente.

16 Me he sentido cómodo/a al solicitar un asesoramiento a mi docente.

17 Me sentí motivado/a a mejorar mi desempeño durante el desarrollo del producto académico de la asignatura.

18 Me siento satisfecho con las competencias adquiridas gracias a la asignatura.

19 Adquirí adecuada comprensión de los contenidos básicos del curso.

20 Tengo la capacidad de describir con claridad los objetivos básicos del curso. 


\begin{tabular}{|c|c|c|c|c|c|c|}
\hline \multirow{2}{*}{$N^{\circ}$} & \multirow{2}{*}{ Ítems } & \multicolumn{5}{|c|}{ Nivel } \\
\hline & & 1 & 2 & 3 & 4 & 5 \\
\hline 21 & Aprendí gran cantidad de contenidos prácticos. & & & & & \\
\hline 22 & Identifico los conceptos más importantes del curso. & & & & & \\
\hline 23 & $\begin{array}{l}\text { El contenido de la asignatura fue de utilidad respecto a mi } \\
\text { futuro ejercicio profesional. }\end{array}$ & & & & & \\
\hline 24 & $\begin{array}{l}\text { He mejorado mis habilidades para el diseño y formulación } \\
\text { de programas preventivos }\end{array}$ & & & & & \\
\hline 25 & $\begin{array}{l}\text { Poseo habilidad para la elaboración de matrices de la fase } \\
\text { de formulación (Árbol de problemas y/o espina de Ishikawa). }\end{array}$ & & & & & \\
\hline 26 & $\begin{array}{l}\text { Aprendí a elaborar instrumentos para la fase de planificación } \\
\text { de programas (Matriz de Marco Lógico). }\end{array}$ & & & & & \\
\hline 27 & $\begin{array}{l}\text { He incrementado mi capacidad para la elaboración de } \\
\text { sesiones de aprendizaje efectivas. }\end{array}$ & & & & & \\
\hline 28 & $\begin{array}{l}\text { He incorporado nuevas estrategias de facilitación de } \\
\text { sesiones de aprendizaje preventivo y/o promocional en } \\
\text { psicología, desde la virtualidad. }\end{array}$ & & & & & \\
\hline 29 & Logré ejecutar el programa que diseñé. & & & & & \\
\hline 30 & $\begin{array}{l}\text { Utilizo estrategias para el monitoreo y evaluación de } \\
\text { programas. }\end{array}$ & & & & & \\
\hline 31 & $\begin{array}{l}\text { Puse en práctica conocimientos básicos en investigación } \\
\text { para la evaluación de los programas ejecutados. }\end{array}$ & & & & & \\
\hline 32 & $\begin{array}{l}\text { Me siento satisfecho con los logros de mi producto } \\
\text { académico (programa diseñado y ejecutado). }\end{array}$ & & & & & \\
\hline
\end{tabular}

